\title{
Synthesis and Luminescence Properties of Nine Novel Carbazolyl Diacylhydrazone Schiff-bases ${ }^{\dagger}$
}

\author{
Limin Wu, Panliang Wu, Dongcai Guo, ${ }^{*}$ Wenqiang Fu, Dong Li, and Tao Luo \\ Chemistry and Chemical Engineering, Hunan University, Changsha 410082, China
}

RECEIVED AUGUST 1, 2013; REVISED MAY 19, 2014; ACCEPTED NOVEMBER 6, 2014

\begin{abstract}
Nine novel carbazolyl diacylhydrazone Schiff-bases were synthesized by alkylation, F-C acylation and condensation reactions starting from carbazole and hydrazide. The title Schiff-bases were characterized by ${ }^{1} \mathrm{H}$ NMR, MS, IR and elemental analysis. The synthetic conditions were optimized, and the best yield of the title Schiff-bases was up to $92.3 \%$. The relationships between the luminescence properties and the structures of the title Schiff-bases were studied. The results showed that the introduction of the Naphthalene-2-yloxy could form great plane conjugate structure to improve their luminescence properties.
\end{abstract}

Keywords: Schiff-bases, carbazole, diacylhydrazone, synthesis, optimize, luminescence property

\section{INTRODUCTION}

As a peculiar Schiff-base, acylhydrazone have received great attention for its strong $\mathrm{p}-\pi$ conjugated system, various coordination forms, strong coordination abilities, good biological activity, ${ }^{1-5}$ catalytic activity ${ }^{6}$ and fluorescence property ${ }^{7,8}$ in recent years. The strong conjugated system and high intramolecular electron transfer efficiency of carbazole make it have potential application prospect in electroluminescence (EL) and photoluminescence (PL). ${ }^{9}$ Carbazole derivatives represent one of the most widespread class of holetransporting materials with strong absorption in the ultraviolet spectral region and blue-light emission. ${ }^{10}$ The conjugated $\pi$ bonds, existed both in acylhydrazone and carbazole, possess a strong ability in energy transfer. However, carbazole has never been used to synthesize the diacylhydrazone Schiff-bases. In this study, we developed nine novel carbazolyl diacylhydrazone Schiff-bases by alkylation, F-C acylation and condensation reactions starting from carbazole and hydrazide. The hydrogen atom of carbonyl and carbazolyl were replaced by several different groups ${ }^{11}$ to clarify the influence of the substituents on the luminescence properties of the Schiff-bases (Scheme 1).

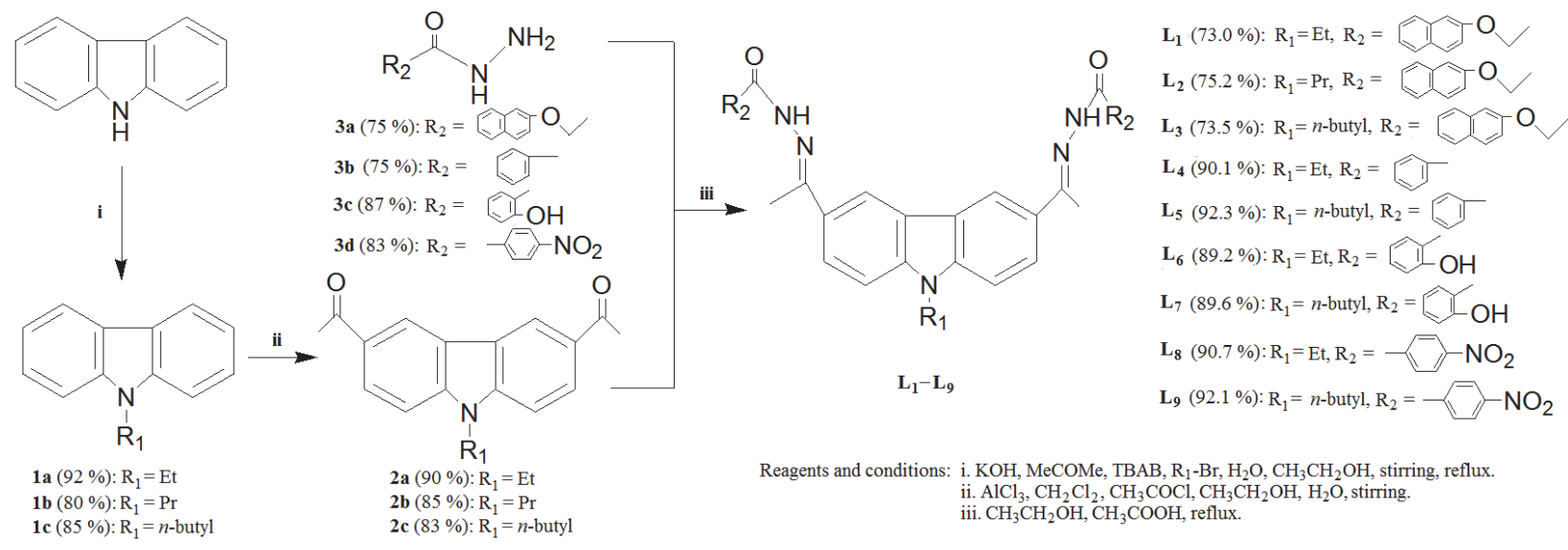

Scheme 1. Synthesis route of carbazolyl diacylhydrazone Schiff-bases.

\footnotetext{
$\dagger$ Dedicated to Professor Mirjana Eckert Maksić on the occasion of her $70^{\text {th }}$ birthday.

* Author to whom correspondence should be addressed. (E-mail: dcguo2001@hnu.edu.cn)
} 


\section{EXPERIMENTAL}

All the chemicals used were of analytical grade and received from commercial suppliers. Solvents were dried before use. Melting points (m.p.) were determined on a XT-4 melting point apparatus (Beijing City) and uncorrected. Elemental analysis $(\mathrm{C}, \mathrm{H}, \mathrm{N})$ data was obtained using a Vario-EI-III elemental analyzer (Gemany). The IR spectra (KBr) were recorded on a PerkinElemer Spectrum One spectrophotometer in the range of 400-4000 $\mathrm{cm}^{-1}$. UV spectra were obtained on a LabTech UV-2100 UV Visible spectrophotometer in the range of 190-450 nm using absolute alcohol as the solvent and internal reference. The $1 \mathrm{H}$ NMR spectra in $\mathrm{CDCl}_{3}$ were recorded on a high resolution NMR spectrometer (Varian-400) using TMS as an internal reference. Florescence spectra were carried out at room temperature on a HITACHI F-2700 fluophotometer.

\section{General Synthetic Procedure of N-alkyl Carbazole (1a-c)}

Carbazole (1.67 g, $10 \mathrm{mmol})$, tetrabutyl ammonium bromide $(0.32 \mathrm{~g}, 1 \mathrm{mmol})$ and potassium hydroxide $(2.24 \mathrm{~g}, 40 \mathrm{mmol})$ dissolved in acetone $(20 \mathrm{~mL})$ were added to a $100 \mathrm{~mL}$ three-necked flask, the mixture solution was stirred for 40 minutes. Alkyl bromide (12 mmol) was added dropwise to the solution with constant stirring. The mixture was refluxed for $1 \mathrm{~h}$. The reaction mixture was then poured into ice water $(300 \mathrm{~mL})$ with vigorous stirring to obtain a great deal of deposit. The mixture was filtered, washed with water and the filter cake obtained was then recrystallized from alcohol and water to give $\mathrm{N}$-alkyl carbazole $(\mathbf{1 a}-\mathbf{1 c}) .^{12}$

$\mathrm{N}$-ethylcarbazole (1a) was synthesised from bromoethane and carbazole. A whitish needle crystals product $(9.2 \mathrm{mmol}, 92 \%)$. m.p $67.0-68.5{ }^{\circ} \mathrm{C}$. MS (EI): $m / z(\%)=152(15), 180(100), 195(\mathrm{M}, 60)$.

N-proplycarbazole (1b) was synthesised from bromopropane and carbazole. A whitish needle crystals product $(8.0 \mathrm{mmol}, 80 \%)$. m.p $49-51{ }^{\circ} \mathrm{C}$. MS (EI): $\mathrm{m} / z(\%)=152(20), 180$ (100), 209 (M, 28).

N-butylcarbazole (1c) was synthesised from bromobutane and carbazole. A whitish needle crystals product $(8.5 \mathrm{mmol}, 85 \%)$. m.p $57.5-59{ }^{\circ} \mathrm{C}$. MS (EI): $m / z(\%)=152(15), 180(100), 223(\mathrm{M}, 30)$.

\section{General Synthetic Procedure of 3,6-Diacetyl-9-alkyl Carbazole (2a-c)}

Aluminium chloride powder $(1.10 \mathrm{~g}, 8 \mathrm{mmol})$ was dissolved partly in dichloromethane $(10 \mathrm{~mL})$ in a $100 \mathrm{~mL}$ three-necked flask with constant stirring until the solution became orange-yellow. To the solution acetyl chloride $(1 \mathrm{~mL}, 10 \mathrm{mmol})$ in dichloromethane and compound 1 ( $2 \mathrm{mmol})$ in dichloromethane was added dropwise to it in an ice bath. The reaction was carried out in the ice bath for $2 \mathrm{~h}$ and further stirred at room temperature for $48 \mathrm{~h}$. The reaction mixture was then poured into ice water $(400 \mathrm{~mL})$ with vigorous stirring to get a pale-yellow precipitate. The crude products were filtered, washed with water, dried, and then recrystallized from alcohol to get compound $2 .^{13}$

3,6-diacetyl-9-ethylcarbazole (2a) was synthesised from acetyl chloride and 1a. A yellow solid (1.80 mmol, $90 \%$ \%. m.p $180-182{ }^{\circ} \mathrm{C}$. MS (EI): $m / z(\%)=221$ (27), 264 (100), 279 (45).

3,6-diacetyl-9-proplycarbazole (2b) was synthesised from acetyl chloride and $\mathbf{1 b}$. A pale-yellow solid (1.70 mmol, $85 \%)$. m.p $165-167{ }^{\circ} \mathrm{C}$. MS (EI): $\mathrm{m} / \mathrm{z}(\%)$ $=279$ (8), $294(\mathrm{M}+1,100), 295$ (20).

3,6-diacetyl-9-butylcarbazole (2c) was synthesised from acetyl chloride and 1c. A pale-yellow solid (1.66 mmol, $83 \%$ ). m.p $127-128^{\circ} \mathrm{C}$. MS (EI): $\mathrm{m} / z(\%)=279$ (5), 308 (M+1, 100), 308 (23).

\section{Synthetic Procedure of Ethyl $\beta$-naphthyloxyacetate}

Ethyl bromoacetate $(5.00 \mathrm{~g}, 30 \mathrm{mmol}), \beta$-naphthol (4.30 g, $30 \mathrm{mmol})$ in acetone $(15 \mathrm{~mL})$ and sodium carbonate anhydrous $(6.20 \mathrm{~g}, 45 \mathrm{mmol})$ were added to a $100 \mathrm{~mL}$ single aperture flask with constant stirring. The solution was refluxed for $14 \mathrm{~h}$ under an anhydrous condition. After standing overnight, the mixture was filtered, washed with cold water $(50 \mathrm{~mL})$ several times, neutralized to $\mathrm{pH} 7-8$ with $10 \% \mathrm{NaOH}$, and extracted with ether $(40 \mathrm{~mL})$ two times. The ether layer collected was dried by sodium sulfate $(4.00 \mathrm{~g})$, and then filtered after standing for $48 \mathrm{~h}$. Then the filtrate was evaporated to remove the ether. The product was not characterized and applied directly to the next step reaction. ${ }^{14}$

\section{Synthetic Procedure of Methyl p-Nitrobenzoate}

$p$-Nitrobenzoic acid (1.67 g, $10 \mathrm{mmol})$ and methanol (10 mmol) were added to a $50 \mathrm{~mL}$ single aperture flask. To the solution concentrated sulphuric acid $(2 \mathrm{~mL})$ was then added dropwise slowly. The mixture was refluxed for $12 \mathrm{~h}$ and poured into ice water, getting lots of white solid. The coarse product was filtered, recrystallized from $50 \%$ alcohol to get a white product, applied directly to the next step reaction. ${ }^{14}$

\section{General Synthetic Procedure of Hydrazide (3a-d)}

Appropriate ester $(10 \mathrm{mmol})$ was dissolved in anhydrous ethanol $(25 \mathrm{~mL})$ in a $100 \mathrm{~mL}$ single aperture flask with constant stirring; $85 \%$ hydrazine hydrate $(3 \mathrm{~mL})$ was added dropwise to it. The solution was heated to reflux for $1 \mathrm{~h}$, cooled down to the room temperature and then diluted with $100 \mathrm{~mL}$ distilled water to get a large white precipitates. The mixture was filtered, washed with 
water, dried, and recrystallized from anhydrous ethanol to get the title compound 3 . $^{14}$

$\beta$-naphthyloxyacethydrazide (3a) was synthesised by the condensation of $85 \%$ hydrazine hydrate with $\beta$-naphthyloxyacetate. A white solid (7.5 mmol, $75 \%)$. m.p $173-175{ }^{\circ} \mathrm{C} .{ }^{1} \mathrm{H}$ NMR (400 MHz, DMSO- $\left.d_{6}\right)$ : $\delta / \mathrm{ppm}=4.37(\mathrm{~s}, 2 \mathrm{H}), 4.61(\mathrm{~s}, 2 \mathrm{H}), 7.24 \sim 7.25(\mathrm{~d}, 1 \mathrm{H}$, $J=4 \mathrm{~Hz}), 7.26 \sim 7.29(\mathrm{t}, 1 \mathrm{H}, J=1.6 \mathrm{~Hz}), 7.35 \sim 7.38(\mathrm{~d}$, $1 \mathrm{H}, J=8 \mathrm{~Hz}), 7.45 \sim 7.49(\mathrm{~d}, 1 \mathrm{H}, J=8.4 \mathrm{~Hz}), 7.77 \sim 7.79$ (d, $1 \mathrm{H}, J=8 \mathrm{~Hz}), 7.84 \sim 7.86(\mathrm{t}, 2 \mathrm{H}, J=3.2 \mathrm{~Hz}), 9.44$ (s, $1 \mathrm{H})$.

Benzoyl hydrazine (3b) was synthesised by the condensation of $85 \%$ hydrazine hydrate with ethyl benzoate. A white solid (7.5 mmol, $75 \%)$. m.p 110.5-113 ${ }^{\circ} \mathrm{C}$. MS (ESI): $m / z(\%)=136(\mathrm{M}, 100), 158$ (46), 159 (5), 235 (20), 277 (6).

Salicylhydrazide (3c) was synthesized by the condensation of $85 \%$ hydrazine hydrate with methyl salicylate. A white solid $(8.7 \mathrm{mmol}, 87 \%)$. m.p 146 147.5 ${ }^{\circ} \mathrm{C}$. MS (ESI): $m / z(\%)=121$ (21), 134 (8), $153(\mathrm{M}+1,100), 154(5), 175$ (10).

$p$-Nitrobenzoylhydrazine (3d) was synthesized by the condensation of $85 \%$ hydrazine hydrate with methyl p-nitrobenzoate. A white solid (8.3 mmol, $83 \%)$. m.p 208-209 ${ }^{\circ} \mathrm{C} .{ }^{1} \mathrm{H}$ NMR $\left(400 \mathrm{MHz}, \mathrm{DMSO}-d_{6}\right): \delta / \mathrm{ppm}=$ $8.21 \sim 8.42(\mathrm{~m}, 4 \mathrm{H}), 9.01(\mathrm{~s}, 1 \mathrm{H}), 4.36(\mathrm{~m}, 2 \mathrm{H})$.

\section{General Synthetic Procedure of the Title Schiff-bases $\left(\mathbf{L}_{1}-\mathbf{L}_{9}\right)$}

The synthesis of Schiff-bases was performed according to the Scheme 1. Compound 2 (1 mmol) and the hydrazide $3(2.2 \mathrm{~mol})$ were dissolved in ethanol $(10 \mathrm{~mL})$ in a $100 \mathrm{~mL}$ three-necked flask, and then glacial acetic acid (2 $\mathrm{mL}$ ) was added dropwise to it. The solution was heated to reflux for $2 \mathrm{~h}$, getting lots of solid. The reaction mixture was then filtered taking the advantage of heat, washed with hot ethanol, and dried to get the title Schiff-base $\mathbf{L}_{\mathbf{1}}-\mathbf{L} \mathbf{9}$.

(Naphthalen-2-yloxy)-acetic Acid \{1-[9-ethyl-6-(1-\{[2(naphthalen-2-yloxy)-acetyl]-hydrazono\}-ethyl)-9Hcarbazol-3-yl]-ethylidene,-hydrazide (L1)

$\mathbf{L}_{\mathbf{1}}$ was synthesized by the condensation of 2a with 3a. A pale-yellow solid $(0.730 \mathrm{mmol}, 73.0 \%)$. m.p 240-242 ${ }^{\circ} \mathrm{C}$. IR (KBr): $v / \mathrm{cm}^{-1}=3416,3055,2966$, 2921, 1693, 1629, 1600, 1531, 1467, 1372, 1258, 1122, 1065, 1065, 952, 748, 701. 1H NMR (400 MHz, DMSO- $\left.d_{6}\right): \delta / \mathrm{ppm}=10.85(\mathrm{~s}, 2 \mathrm{H}, \mathrm{NH}), 8.72(\mathrm{t}, 2 \mathrm{H}, J=$ $15 \mathrm{~Hz}, \mathrm{ArH}), 8.09$ (q, 2H, $J=9 \mathrm{~Hz}, \mathrm{ArH}), 7.84$ (m, 6H, ArH), 7.67 (t, 2H, $J=8.5 \mathrm{~Hz}, \operatorname{ArH}), 7.45$ (q, 2H, $J=$ $9 \mathrm{~Hz}, \mathrm{ArH}), 7.34$ (m, 6H, ArH), $5.38\left(\mathrm{~m}, 2 \mathrm{H}, \mathrm{CH}_{2}\right), 4.90$ (s, 2H, $\left.\mathrm{CH}_{2}\right), 4.49$ (q, 2H, $\left.J=6 \mathrm{~Hz}, \mathrm{CH}_{2}\right), 2.49$ (s, 6H, $\left.\mathrm{N}=\mathrm{C}-\mathrm{CH}_{3}\right), 1.33\left(\mathrm{t}, 3 \mathrm{H}, J=7.5 \mathrm{~Hz}, \mathrm{CH}_{3}\right) . \mathrm{MS}(\mathrm{ESI})$ : $m / z(\%)=1373(2 \mathrm{M}+\mathrm{Na}+, 100), 1174$ (22), 770 (25), $698\left(\mathrm{M}+\mathrm{Na}^{+}, 46\right), 235$ (27). Anal. Calcd for
$\mathrm{C}_{42} \mathrm{H}_{37} \mathrm{~N}_{5} \mathrm{O}_{2}$ : C, 74.65; H, 5.52; N, 10.30. Found: C, 74.63; H, 5.74; N, 10.26.

(Naphthalen-2-yloxy)-acetic Acid \{1-[6-(1-\{[2(naphthalen-2-yloxy)-acetyl]-hydrazono\}-ethyl)-9propyl-9H-carbazol-3-yl]-ethylidene $\}$-hydrazide $\left(\boldsymbol{L}_{2}\right)$ $\mathbf{L}_{\mathbf{2}}$ was synthesized by the condensation of $\mathbf{2} \mathbf{b}$ with $\mathbf{3 a}$. A pale-yellow solid $(0.752 \mathrm{mmol}, 75.2 \%)$. m.p 236-238 ${ }^{\circ} \mathrm{C}$. IR (KBr): $v / \mathrm{cm}^{-1}=3443,3055,2955$, 2922, 2853, 1661, 1628, 1600, 1549, 1470, 1256, 1122, 1072, 1065, 952, 739, 701. ${ }^{1} \mathrm{H}$ NMR (400 MHz, DMSO- $\left.d_{6}\right): \delta / \mathrm{ppm}=10.81(\mathrm{~s}, 2 \mathrm{H}, \mathrm{NH}), 8.75(\mathrm{~d}, 2 \mathrm{H}, J=$ $4 \mathrm{~Hz}, \mathrm{ArH}), 8.15$ (q, 2H, $J=3.6 \mathrm{~Hz}, \mathrm{ArH}), 7.85$ (m, 6H, ArH), 7.65 (d, 2H, $J=4 \mathrm{~Hz}, \operatorname{ArH}), 7.42$ (m, 2H, ArH), 7.31 (m, 6H, ArH), $5.38\left(\mathrm{~m}, 2 \mathrm{H}, \mathrm{CH}_{2}\right), 4.42(\mathrm{~m}, 2 \mathrm{H}$, $\left.\mathrm{CH}_{2}\right), 2.40\left(\mathrm{~s}, 6 \mathrm{H}, \mathrm{N}=\mathrm{C}-\mathrm{CH}_{3}\right), 1.82\left(\mathrm{~s}, 4 \mathrm{H}, \mathrm{CH}_{2}\right), 0.86$ $\left(\mathrm{t}, 3 \mathrm{H}, \mathrm{CH}_{3}\right)$. MS (EI): $m / z(\%)=690(\mathrm{M}+1,8), 689(\mathrm{M}$, 12), 490 (11), 262 (13), 201 (13), 144 (100). Anal. Calcd for $\mathrm{C}_{43} \mathrm{H}_{39} \mathrm{~N}_{5} \mathrm{O}_{2}$ : C, 74.87; H, 5.70; N, 10.15 . Found: C, 74.53; H, 5.60; N, 9.84.

(Naphthalen-2-yloxy)-acetic Acid \{1-[9-butyl-6-(1-\{[2(naphthalen-2-yloxy)-acetyl]-hydrazono\}-ethyl)-9H-

carbazol-3-yl]-ethylidene, -hydrazide $\left(\boldsymbol{L}_{3}\right)$

$\mathbf{L}_{\mathbf{3}}$ was synthesized by the condensation of $\mathbf{2 c}$ with $\mathbf{3 a}$. A pale-yellow solid $(0.735 \mathrm{mmol}, 73.5 \%)$. m.p 230-232 ${ }^{\circ} \mathrm{C}$. IR (KBr): $v / \mathrm{cm}^{-1}=3415,3054,2955$, 2927, 2872, .1683, 1629, 1599, 1509, 1466, 1255, 1122, 1070, 1070, 961, 744, 700. ${ }^{1} \mathrm{H}$ NMR (400 MHz, DMSO- $\left.d_{6}\right): \delta / \mathrm{ppm}=10.85(\mathrm{~s}, 2 \mathrm{H}, \mathrm{NH}), 8.74(\mathrm{~d}, 2 \mathrm{H}$, $J=11 \mathrm{~Hz}, \mathrm{ArH}), 8.11$ (d, 2H, $J=6 \mathrm{~Hz}, \mathrm{ArH}), 8.04$ (t, $2 \mathrm{H}, J=7.5 \mathrm{~Hz}, \mathrm{ArH}), 7.85(\mathrm{~m}, 4 \mathrm{H}, \mathrm{ArH}), 7.71(\mathrm{t}, 2 \mathrm{H}$, $J=9 \mathrm{~Hz}, \operatorname{ArH}), 7.65$ (d, 2H, $J=9 \mathrm{~Hz}, \mathrm{ArH}), 7.43$ (t, $4 \mathrm{H}, J=7.5 \mathrm{~Hz}, \mathrm{ArH}), 7.34(\mathrm{t}, 2 \mathrm{H}, J=6.5 \mathrm{~Hz}, \mathrm{ArH})$, $5.38\left(\mathrm{~m}, 2 \mathrm{H}, \mathrm{CH}_{2}\right), 4.45\left(\mathrm{~m}, 2 \mathrm{H}, J=6 \mathrm{~Hz}, \mathrm{CH}_{2}\right), 2.50(\mathrm{~s}$, $\left.6 \mathrm{H}, \mathrm{N}=\mathrm{C}-\mathrm{CH}_{3}\right), 1.78\left(\mathrm{t}, 2 \mathrm{H}, J=7.5 \mathrm{~Hz}, \mathrm{CH}_{2}\right), 1.27(\mathrm{~s}$, $\left.4 \mathrm{H}, J=7.5 \mathrm{~Hz}, \mathrm{CH}_{2}\right), 0.88\left(\mathrm{t}, 3 \mathrm{H}, \mathrm{CH}_{3}\right) . \mathrm{MS}(\mathrm{EI})$ : $m / z(\%)=704(\mathrm{M}+1,12), 703(\mathrm{M}, 23), 546(11), 262(8)$, 201 (13), 144 (100). Anal. Calcd for $\mathrm{C}_{44} \mathrm{H}_{41} \mathrm{~N}_{5} \mathrm{O}_{2}$ : C, 75.09; H, 5.87; N, 9.95. Found: C, 74.92; H, 6.10; N, 9.93 .

Benzoic Acid \{1-[9-ethyl-6-(1-\{[2-(phenyl)-formacyl]hydrazono\}-ethyl)-9H-carbazol-3-yl]-ethylidene\}hydrazide $\left(\boldsymbol{L}_{4}\right)$

$\mathbf{L}_{4}$ was synthesized by the condensation of $\mathbf{2 a}$ with $\mathbf{3 b}$. A pale-yellow solid (0.901 mmol, $90.1 \%)$. m.p 314-315 ${ }^{\circ} \mathrm{C}$. IR (KBr): $v / \mathrm{cm}^{-1}=3414,3237,1638$, 1617, 1528, 1484, 1399, 1284, 1132, 718. ${ }^{1} \mathrm{H}$ NMR $\left(400 \mathrm{MHz}, \mathrm{DMSO}-d_{6}\right): \delta / \mathrm{ppm}=10.76(\mathrm{~s}, 2 \mathrm{H}, \mathrm{NH})$, $8.75(\mathrm{~s}, 2 \mathrm{H}, \mathrm{ArH}), 8.11(\mathrm{~m}, 2 \mathrm{H}, \mathrm{ArH}), 7.92$ (s, 4H, $\operatorname{ArH}), 7.72$ (q, 2H, $J=9 \mathrm{~Hz}, \operatorname{ArH}), 7.59$ (q, 2H, $J=$ $2.5 \mathrm{~Hz}, \mathrm{ArH}), 7.53(\mathrm{~m}, 4 \mathrm{H}, \mathrm{ArH}), 4.51\left(\mathrm{~m}, 2 \mathrm{H}, \mathrm{CH}_{2}\right)$, $2.50\left(\mathrm{~s}, 6 \mathrm{H}, \mathrm{N}=\mathrm{C}-\mathrm{CH}_{3}\right), 1.35\left(\mathrm{t}, 3 \mathrm{H}, J=2 \mathrm{~Hz}, \mathrm{CH}_{3}\right)$. MS (EI): $m / z(\%)=516(\mathrm{M}+1,14), 515(\mathrm{M}, 43), 397$ (33), 292 (24), 250 (24), 105 (100). Anal. Calcd for 
$\mathrm{C}_{32} \mathrm{H}_{29} \mathrm{~N}_{5} \mathrm{O}_{2}$ : C, 74.54; $\mathrm{H}$, 5.67; N, 13.58. Found: C, 74.18; H, 5.65; N, 13.26 .

Benzoic Acid \{1-[9-butyl-6-(1-\{[2-(phenyl)-formacyl]hydrazono\}-ethyl)-9H-carbazol-3-yl] - ethylidene\}hydrazide $\left(\boldsymbol{L}_{5}\right)$

L5 was synthesized by the condensation of $\mathbf{2 c}$ with $\mathbf{3 b}$. A pale-yellow solid $(0.923 \mathrm{mmol}, 92.3 \%)$. m.p 262-263 ${ }^{\circ} \mathrm{C}$. IR (KBr): $v / \mathrm{cm}^{-1}=3421,3134,1637$, 1606, 1523, 1485, $1399,1284,1132,720 .{ }^{1} \mathrm{H}$ NMR $\left(400 \mathrm{MHz}, \mathrm{DMSO}-d_{6}\right): \delta / \mathrm{ppm}=10.77(\mathrm{~s}, 2 \mathrm{H}, \mathrm{NH})$, 8.74 (s, 2H, ArH), $8.11(\mathrm{~m}, 2 \mathrm{H}, \mathrm{ArH}), 7.91$ (d, 4H, $J=$ $5.5 \mathrm{~Hz}, \mathrm{ArH}), 7.69$ (m, 2H, ArH), 7.54 (m, 2H, ArH), 7.45 (m, 4H, ArH), 4.46 (t, 2H, J= $7 \mathrm{~Hz}, \mathrm{CH}_{2}$ ), 2.69 (m, $\left.2 \mathrm{H}, \mathrm{CH}_{2}\right), 2.49\left(\mathrm{~s}, 6 \mathrm{H}, \mathrm{N}=\mathrm{C}-\mathrm{CH}_{3}\right), 1.77\left(\mathrm{~m}, 2 \mathrm{H}, \mathrm{CH}_{2}\right)$, $0.86\left(\mathrm{~m}, 3 \mathrm{H}, \mathrm{CH}_{3}\right)$. MS (EI): $m / z(\%)=544(\mathrm{M}+1,35)$, 543 (M, 91), 424 (21), 320 (18), 289 (22), 205 (21), 105 (100). Anal. Calcd for $\mathrm{C}_{34} \mathrm{H}_{33} \mathrm{~N}_{5} \mathrm{O}_{2}$ : C, 75.11; H, 6.12; N, 12.88. Found: C, 74.79; H, 5.95; N, 13.03.

Salicylic Acid \{1-[9-ethyl-6-(1-\{[2-(2-hydroxyphenyl)formacyl]-hydrazono\}-ethyl) - 9H-carbazol-3-yl]ethylidene,-hydrazide $\left(\boldsymbol{L}_{6}\right)$

$\mathbf{L}_{\mathbf{6}}$ was synthesized by the condensation of $\mathbf{2 a}$ with $\mathbf{3 c}$. A pale-yellow solid $(0.892 \mathrm{mmol}, 89.2 \%)$. m.p 236-237 ${ }^{\circ} \mathrm{C}$. IR (KBr): $v / \mathrm{cm}^{-1}=3547,3477,3415$, 3274, 2966, 2928, 1638, 1618, 1602, 1455, 1562, 1486, 1275, 1153, 1099, 748. ${ }^{1} \mathrm{H}$ NMR (400 MHz DMSO- $\left.d_{6}\right)$ : $\delta / \mathrm{ppm}=11.84(\mathrm{~s}, 2 \mathrm{H}, \mathrm{OH}), 11.34(\mathrm{~s}, 2 \mathrm{H}, \mathrm{NH}), 8.75(\mathrm{~s}$, 2H, ArH), 8.14 (d, 2H, $J=8.5 \mathrm{~Hz}, \mathrm{ArH}), 8.03$ (d, 2H, $J=8 \mathrm{~Hz}, \operatorname{ArH}), 7.72(\mathrm{~d}, 2 \mathrm{H}, J=8.5 \mathrm{~Hz}, \operatorname{ArH}), 7.43$ (q, $2 \mathrm{H}, J=7 \mathrm{~Hz}, \mathrm{ArH}), 7.03(\mathrm{~m}, 4 \mathrm{H}, \mathrm{ArH}), 4.51$ (d, 2H, $J=$ $\left.7 \mathrm{~Hz}, \mathrm{CH}_{2}\right), 2.50\left(\mathrm{~s}, 6 \mathrm{H}, \mathrm{N}=\mathrm{C}-\mathrm{CH}_{3}\right), 1.37$ (t, 3H, $J=$ $\left.6.5 \mathrm{~Hz}, \mathrm{CH}_{3}\right)$. MS (EI): $m / z(\%)=548(\mathrm{M}+1,3), 547$ (M, 7), 426 (9), 152 (18), 121 (100), 92 (44), 65 (26). Anal. Calcd for $\mathrm{C}_{32} \mathrm{H}_{29} \mathrm{~N}_{5} \mathrm{O}_{4}: \mathrm{C}, 70.19 ; \mathrm{H}, 5.34 ; \mathrm{N}$, 12.79. Found: C, 70.40; H, 5.25; N, 12.91 .

Salicylic Acid \{1-[9-butyl-6-(1-\{[2-(2-hydroxyphenyl)formacyl]-hydrazono\}-ethyl) - 9H-carbazol-3-yl]ethylidene -hydrazide $\left(\boldsymbol{L}_{7}\right)$

$\mathbf{L}_{7}$ was synthesized by the condensation of $\mathbf{2 c}$ with $\mathbf{3 c}$. A pale-yellow solid $(0.896 \mathrm{mmol}, 89.6 \%)$. m.p 257-258 ${ }^{\circ} \mathrm{C}$. IR (KBr): $v / \mathrm{cm}^{-1}=3547,3476,3413$, 3231, 2962, 2933, 1637, 1617, 1559, 1487, 1455, 1277, 1154, 1100, 754. ${ }^{1} \mathrm{H}$ NMR (400 MHz, DMSO- $\left.d_{6}\right)$ : $\delta / \mathrm{ppm}=11.83(\mathrm{~s}, 2 \mathrm{H}, \mathrm{OH}), 11.34(\mathrm{~s}, 2 \mathrm{H}, \mathrm{NH}), 8.74(\mathrm{~s}$, $2 \mathrm{H}, \mathrm{ArH}), 8.12$ (d, $2 \mathrm{H}, J=8.5 \mathrm{~Hz}, \mathrm{ArH}), 8.03$ (d, 2H, $J=6 \mathrm{~Hz}, \operatorname{ArH}), 7.80(\mathrm{~d}, 2 \mathrm{H}, J=1.5 \mathrm{~Hz}, \mathrm{ArH}), 7.78(\mathrm{~d}$, $2 \mathrm{H}, J=1.5 \mathrm{~Hz}, \operatorname{ArH}), 7.71(\mathrm{~d}, 2 \mathrm{H}, J=8.5 \mathrm{~Hz}, \mathrm{ArH})$, $7.37(\mathrm{t}, 2 \mathrm{H}, J=8.5 \mathrm{~Hz}, \mathrm{ArH}), 4.47\left(\mathrm{~s}, 2 \mathrm{H}, \mathrm{CH}_{2}\right), 2.50(\mathrm{~s}$, $\left.6 \mathrm{H}, \mathrm{N}=\mathrm{C}-\mathrm{CH}_{3}\right), 1.81\left(\mathrm{q}, 2 \mathrm{H}, J=7 \mathrm{~Hz}, \mathrm{CH}_{2}\right), 1.33(\mathrm{q}$, $\left.2 \mathrm{H}, J=7.5 \mathrm{~Hz}, \mathrm{CH}_{2}\right), 0.89\left(\mathrm{t}, 3 \mathrm{H}, J=7.5 \mathrm{~Hz}, \mathrm{CH}_{3}\right)$. MS (EI) $m / z(\%): 576(\mathrm{M}+1,7), 575$ (M, 12), 424(17), 319(12), 262 (12), 137(24), 121(100). Anal. Calcd for $\mathrm{C}_{34} \mathrm{H}_{33} \mathrm{~N}_{5} \mathrm{O}_{4}$ : C, 70.94; H, 5.78; N, 12.17. Found: C, 70.76; H, 5.84; N, 12.17 .
4-Nitro-benzoic Acid \{1-[9-ethyl-6-(1-\{[2-(4nitrophenyl)-formacyl]-hydrazono\}-ethyl)-9H-carbazol3-yl]-ethylidene,-hydrazide $\left(\boldsymbol{L}_{8}\right)$

$\mathbf{L}_{\mathbf{8}}$ was synthesized by the condensation of $\mathbf{2 a}$ with $\mathbf{3 d}$. A orange-yellow solid $(0.907 \mathrm{mmol}, 90.7 \%)$. m.p $312-313{ }^{\circ} \mathrm{C}$. IR (KBr): $v / \mathrm{cm}^{-1}=3479,3413,3231$, $1638,1617,1520,1487,1400,1345,1277,1133,860$. ${ }^{1} \mathrm{H}$ NMR (400 MHz, DMSO- $\left.d_{6}\right): \delta / \mathrm{ppm}=11.10(\mathrm{~s}, 2 \mathrm{H}$, $\mathrm{NH}), 8.83$ (s, 2H, ArH), 8.38 (d, 6H, $J=9 \mathrm{~Hz}$, ArH), $8.16(\mathrm{~d}, 2 \mathrm{H}, J=10.5 \mathrm{~Hz}, \operatorname{ArH}), 7.72(\mathrm{~d}, 2 \mathrm{H}, J=9.5 \mathrm{~Hz}$, ArH), 7.60 (m, 2H, ArH), $4.53\left(\mathrm{~m}, 2 \mathrm{H}, \mathrm{CH}_{2}\right), 2.51$ (s, $\left.6 \mathrm{H}, \mathrm{N}=\mathrm{C}-\mathrm{CH}_{3}\right), 1.36\left(\mathrm{t}, 3 \mathrm{H}, J=6 \mathrm{~Hz}, \mathrm{CH}_{3}\right) . \mathrm{MS}(\mathrm{EI})$ : $m / z(\%)=606(\mathrm{M}+1,3), 605(\mathrm{M}, 6), 263$ (14), $166(42)$, 150 (100), 120 (76), 92 (46). Anal. Calcd for $\mathrm{C}_{32} \mathrm{H}_{27} \mathrm{~N}_{7} \mathrm{O}_{6}$ : C, 63.46; H, 4.49; N, 16.19. Found: C, $63.71 ; \mathrm{H}, 4.88 ; \mathrm{N}, 15.86$.

\section{4-Nitro-benzoic Acid \{1-[9-butyl-6-(1-\{[2-(4-} nitrophenyl)-formacyl]-hydrazono\}-ethyl)-9H-carbazol3-yl]-ethylidene,-hydrazide ( $\left.\boldsymbol{L}_{\boldsymbol{g}}\right)$

L9 was synthesized by the condensation of $\mathbf{2 c}$ with $\mathbf{3 d}$. A yellow solid (0.921 mmol, $92.1 \%$ ). m.p 295-296 ${ }^{\circ} \mathrm{C}$. IR (KBr): $v / \mathrm{cm}^{-1}=3476,3414,3237,2957,2928$, 2870, 1639, 1617, 1600, 1526, 1485, 1344, 1285, 1139, 1109, 864. ${ }^{1} \mathrm{H}$ NMR (400 MHz, DMSO- $\left.d_{6}\right): \delta / \mathrm{ppm}=$ 11.10 (s, 2H, NH), 8.78 (s, 2H, ArH), 8.38 (d, 4H, $J=$ $8.5 \mathrm{~Hz}, \mathrm{ArH}), 8.18$ (d, 4H, $J=9 \mathrm{~Hz}, \mathrm{ArH}), 8.13$ (d, 2H, $J=5.5 \mathrm{~Hz}, \mathrm{ArH}), 7.73(\mathrm{t}, 2 \mathrm{H}, J=9 \mathrm{~Hz}, \mathrm{ArH}), 4.49(\mathrm{~m}$, $\left.2 \mathrm{H}, \mathrm{CH}_{2}\right), 2.51\left(\mathrm{t}, 6 \mathrm{H}, J=2 \mathrm{~Hz}, \mathrm{~N}=\mathrm{C}-\mathrm{CH}_{3}\right), 1.79(\mathrm{~m}$, $\left.2 \mathrm{H}, J=5.5 \mathrm{~Hz}, \mathrm{CH}_{2}\right), 1.32\left(\mathrm{~m}, 2 \mathrm{H}, \mathrm{CH}_{2}\right), 0.90$ (t, $3 \mathrm{H}$, $\left.\mathrm{CH}_{3}\right)$. MS (EI): $m / z(\%)=633(\mathrm{M}, 2), 247$ (5), $166(45)$, 150 (100), 120 (31), 104 (40), 76 (26). Anal. Calcd for $\mathrm{C}_{34} \mathrm{H}_{31} \mathrm{~N}_{7} \mathrm{O}_{6}$ : C, 64.45; H, 4.97; N, 15.47. Found: C, 64.12; H, 5.05; N, 15.08 .

\section{RESULTS AND DISCUSSION}

\section{Optimized Synthetic Conditions of the Title Schiff- bases}

The yields of the title carbazolyl diacylhydrazone Schiff-bases in the condensation reactions between 3,6-diacetyl-9-alkylcarbazole and hydrazide were found to be highly sensitive to the acid catalyst (glacial acetic acid) and the reaction time. The synthetic conditions of benzoic acid \{1-[9-butyl-6-(1-\{[2-(phenyl)formacyl]-hydrazono -ethyl)-9H-carbazol-3-yl]-ethylidene\}-hydrazide (L5) were optimized and expected to be helpful to the synthesis of other title Schiffbases.

Because the glacial acetic acid can enhance the electropositivity of the carbonyl carbon, promote the breakdown of the carbazolyl diacylhydrazone Schiffbases, and reduce the nucleophilic ability of the amino, the condensation reactions were sensitive to the acid catalyst (glacial acetic acid). 
Table 1. The yields of the title Schiff-bases

\begin{tabular}{ccc}
\hline Schiff-base & Yield $^{(\mathrm{a})} / \%$ & Yield $^{(\mathrm{b})} / \%$ \\
\hline $\mathbf{L} \mathbf{1}$ & 10.1 & 73.0 \\
$\mathbf{L}_{\mathbf{2}}$ & 11.4 & 75.2 \\
$\mathbf{L} 3$ & 12.5 & 73.5 \\
$\mathbf{L} \mathbf{4}$ & 10.3 & 90.1 \\
$\mathbf{L}$ 5 & 11.8 & 92.3 \\
$\mathbf{L} 6$ & 13.4 & 89.2 \\
$\mathbf{L}$ 7 & 10.6 & 89.6 \\
$\mathbf{L} 8$ & 12.9 & 90.7 \\
$\mathbf{L 9}$ & 10.4 & 92.1 \\
\hline
\end{tabular}

(a) The yields of $\mathbf{L}_{1}-\mathbf{L} 9$ in the absence of glacial acetic acid.

(b) The yields of $\mathbf{L}_{\mathbf{1}}-\mathbf{L} \mathbf{9}$ in glacial acetic acid.

In order to investigate the influence of the acid catalyst on the yields of the title Schiff-bases, the condensation reactions of 3,6-diacetyl-9-alkylcarbazole with hydrazide were carried out in the presence of glacial acetic acid or not. The experimental data were listed in Table 1. It is seen from the table 1 that the yields of the title Schiff-bases were no more than $15 \%$ in the absence of glacial acetic acid. However, the yields of $\mathbf{L}_{4}-\mathbf{L} 9$ were all about $90 \%$ in the presence of glacial acetic acid. The slightly lower yields of $\mathbf{L}_{\mathbf{1}}-\mathbf{L}_{\mathbf{3}}$ may be due to the steric hindrance of naphthalene-2-yloxy. The results indicated that the addition of the glacial acetic acid and the steric hindrance from the substituents in benzene ring had remarkable influence on the yields of the title Schiff-bases, and the electronic effect of the substituents had little influence on them.

In order to optimize the synthetic conditions of the title Schiff-bases, the influences of the reaction time and the volumn fraction of glacial acetic acid on the yield of L5 were investigated. The experimental data were listed in Table 2.

Based on the optimization experimental data in Table 2, it was concluded that the optimal synthesis conditions of the compound $\mathbf{L}_{\mathbf{5}}$ via the condensation of benzoylhydrazine with 3, 6-diacetyl-9- butylcarbazole were that the volumn fraction of glacial acetic acid was $20 \%$ and the reaction time was $2 \mathrm{~h}$, the yield of the

Table 2. The yields of the Schiff-base $\mathbf{L}_{5}$

\begin{tabular}{cccc}
\hline Entry & vol. $^{(\text {a) } / \%}$ & $t / \mathrm{h}$ & Yield/\% \\
\hline 1 & 0 & 1 & 0 \\
2 & 3 & 1 & 45.1 \\
3 & 10 & 1 & 74.5 \\
4 & 10 & 2 & 85.6 \\
5 & 20 & 2 & 92.3 \\
6 & 30 & 2 & 92.3 \\
\hline
\end{tabular}

(a) The volume fraction of glacial acetic acid.
Table 3. The UV spectra data of the title Schiff-bases $\mathbf{L}_{\mathbf{1}}-\mathbf{L} \mathbf{9}$

\begin{tabular}{ccc}
\hline Schiff-base & $\lambda_{\mathrm{a}, 1} / \mathrm{nm}$ & $\lambda_{\mathrm{a}, 2} / \mathrm{nm}$ \\
\hline $\mathbf{L}_{1}$ & 302 & 330 \\
$\mathbf{L}_{\mathbf{2}}$ & 317 & 348 \\
$\mathbf{L}_{3}$ & 310 & 332 \\
$\mathbf{L}_{\mathbf{4}}$ & 308 & 340 \\
$\mathbf{L}_{\mathbf{5}}$ & 290 & 320 \\
$\mathbf{L}_{6}$ & 297 & 326 \\
$\mathbf{L}_{7}$ & 266 & 307 \\
$\mathbf{L}_{\mathbf{3}}$ & 268 & 310 \\
$\mathbf{L} 9$ & 277 & 312 \\
\hline
\end{tabular}

compound $\mathbf{L}_{5}$ was up to $92.3 \%$ under this reaction condition.

\section{Effect of Substituents on the Properties of the Title Schiff-bases}

All the title Schiff-bases showed the luminescence properties, however, their fluorescent intensities were quite different. In order to evaluate the relationships between the luminescence properties and the structures of the title Schiff-bases, the UV spectra and fluorescence emission spectra of the title Schiff-bases were measured.

The UV spectra data of the title Schiff-bases ( $\mathbf{L}_{1}-$ L9) were listed in Table 3 and the UV spectra were shown in Figure 1 and Figure 2. The UV absorption bands of $\mathbf{L}_{1}-\mathbf{L} 9$ appeared at $\lambda_{\mathrm{a}, 1}$ and $\lambda_{\mathrm{a}, 2}$ were assigned to the $\pi-\pi^{*}$ and $n-\pi^{*}$ transition respectively.

The fluorescence emission spectra of the title Schiff-bases $\left(\mathbf{L}_{1}-\mathbf{L} \mathbf{9}\right)$ were excitated by their respective maximum UV absorption peak wavelengths.

The fluorescence emission spectra of the compounds $\mathbf{L}_{1}-\mathbf{L}_{3}$ were shown in Figure 3. It was found that the fluorescence emission intensities of the compounds $\mathbf{L}_{1}$ and $\mathbf{L}_{2}$ were strong due to the introduction of naphthalene-2-yloxy which could improve the structural planarity and conjugation effect of the compounds $\mathbf{L}_{\mathbf{1}}$ and $\mathbf{L}_{2}$. However, when a butyl group was bonded to the carbazole ring, such as the compound $\mathbf{L}_{3}$, the fluorescence emission intensity was observed to be much weaker relative to that of the compounds $\mathbf{L}_{1}$ and $\mathbf{L}_{2}$ because the increase of alkyl carbon numbers in the carbazole ring resulted in the reduction of the planarity of the whole molecular structure. In addition, the title Schiff-bases with benzene ring, such as the compounds $\mathbf{L}_{\mathbf{4}}-\mathbf{L}$, , showed particularly weak fluorescence because of their poor conjugated structure. The experimental results clearly indicated that both the naphthalene-2yloxy structure and the change of the alkyl group in the carbazole ring had remarkable influence on the luminescence properties of the title Schiff-bases. 


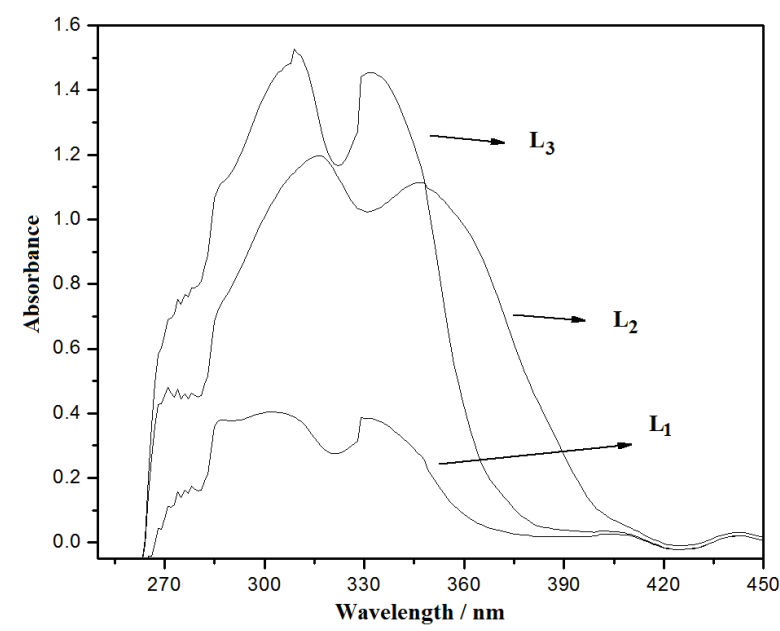

Figure 1. UV spectra of the Schiff-bases $\left(\mathbf{L}_{1}-\mathbf{L}_{3}\right)$.

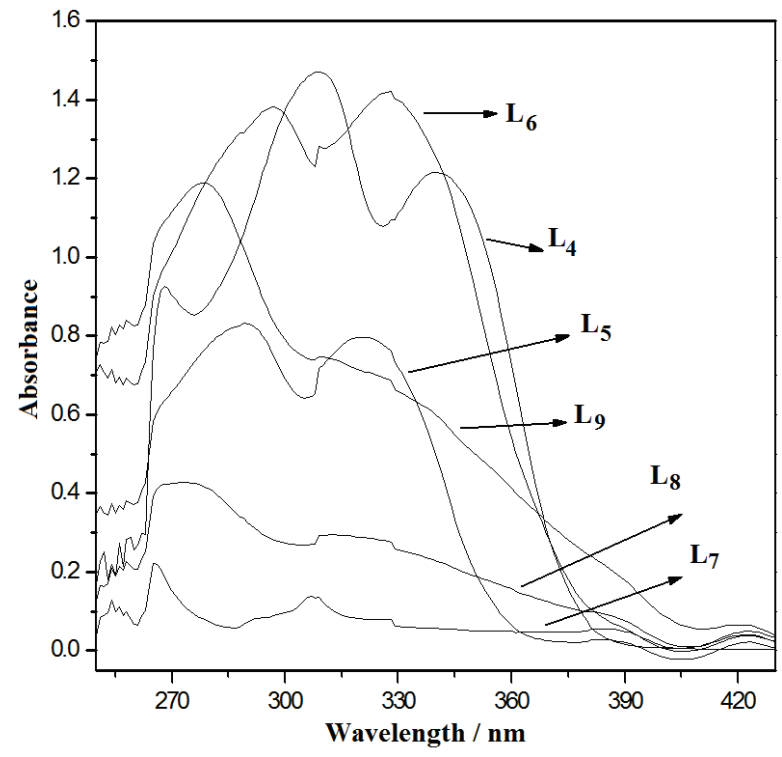

Figure 2. UV spectra of the Schiff-bases $\left(\mathbf{L}_{\mathbf{4}}-\mathbf{L}_{\mathbf{9}}\right)$.

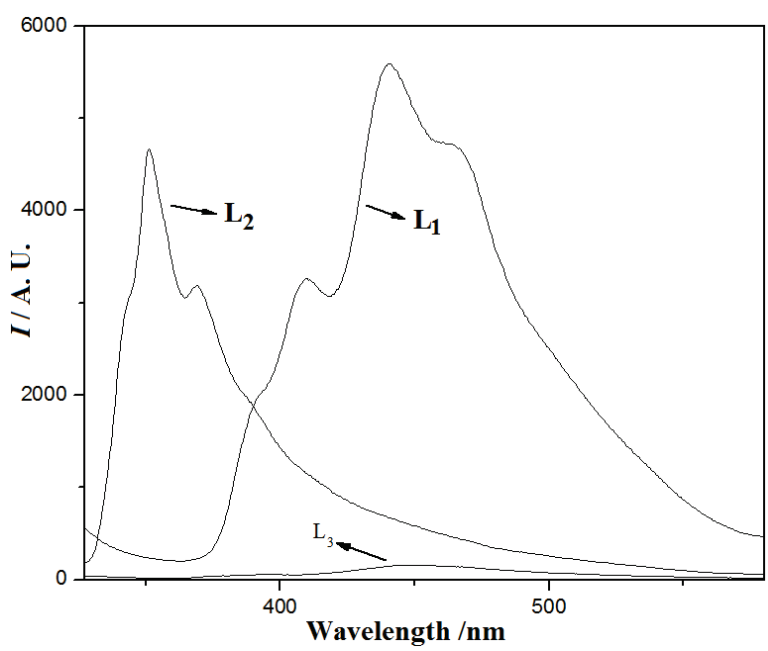

Figure 3. Fluorescence emission spectra of the Schiff-bases $\left(\mathbf{L}_{1}-\mathbf{L}_{3}\right)$.

\section{CONCLUSION}

In conclusion, an efficient synthetic approach to nine novel carbazolyl diacylhydrazone Schiff-bases obtained from carbazole and hydrazide by alkylation and FriedelCrafts acylation have been developed. All synthesized compounds were examined by ${ }^{1} \mathrm{H}$ NMR, MS, IR, elemental analysis and so on. The yield of the title Schiffbases rise up to $92.3 \%$ under the optimized synthetic conditions that the volumn fraction of glacial acetic acid was $20 \%$ and the reaction time was $2 \mathrm{~h}$. The relationship between luminescence properties and the structures of the title Schiff-bases were studied by introducing naphthalene-2-yloxy, benzene ring and alkyl. It was observed that the introduction of naphthalene-2-yloxy causes the fluorescence emission intensity of the title Schiff-bases to be increased considerably. But the electronic effects of benzene ring and the substituents in benzene ring barely affect the luminous intensity of the title Schiff-bases.

Acknowledgements. The authors are grateful for the financial support of the National Natural Science Foundation of China (No. J1103312, No.21341010), the Innovative Research Team in University (No.IRT1238) as well as the Science and Technology Project of Hunan provincial Science and Technology Department (No.2012GK3156). We also thank Dr. William Hickey, the U.S. professor of HRM, for the English editing on this paper.

\section{REFERENCES}

1. M. Katyal and Y. Dutt, Talanta 22 (1975) 151-166.

2. R. N. Sharma, K. P.Sharma, and S. N. Dikshit, J. Adv. Sci. Res. 2 (2011) 5-10

3. S. M. Xiao, Asian J. Chem. 21 (2009) 4927-4931.

4. D. R. Richardson and P. V. Bernhardt, J. Biol. Inorg. Chem. 4 (1999) 266-273.

5. P. V. Bernhardt, P. Chin, P.C. Sharpe, and D. R. Richardson, J. Chem. Soc. Dalton Trans. 36 (2007) 3232-3234.

6. H. H. Monfared, S. Kheirabadi, N. A. Lalami, and P. Mayer, Polyhedron 30 (2011) 1375-1384.

7. K. C. Majumdar, B. Chattopadhyay, S. Chakravorty, N. Pal, and R.K. Sinha, Tetrahedron Lett. 49 (2008) 7149-7152.

8. S. Mukherjee, S. Chowdhury, A. P. Chattapadhyay, and B. Arindam, Inorg. Chim. Acta 373 (2011) 40-46.

9. D. C. Guo, P. L. Wu, H. Tan, L. Xia, and W. H. Zhou, J. Lumin. 131 (2011) 1272-1276.

10. G. Bubniene, M. Daskeviciene, A. Pukalskas, V. Jankayskas, and V. Getaustis, Molecules 17 (2012) 14846-14857.

11. M. Kirkus, J. Simokaitiene, J. V. Grazulevicius, and V. Jankauskas, Synthetic Met. 160 (2010) 750-755.

12. L. Li, P. Wang, Y. C. Zhang, Y. Q. Wu, Z. M. Chen, and C. Y. He, J. Mol. Struct. 1051 (2013) 23-29.

13. T. Valta, E. M. Puputti, L. Huppanen, J. Kankare, H. Takalo, and T. Soukka, Anal. Chem. 84 (2012) 7708-7712.

14. L. Maingot, J. Eibakali, J. Dumont, D. Bosc, N. Cousaert, A. Urban, G. Deglane, B. Villoutreix, H. Nagase, O. Sperandio, F. Leroux, B. Deprez, and R. D. Poulain, Eur. J. Med. Chem. 69 (2013) 244-261. 